\title{
Randomized comparison of popliteal-sciatic perineural catheter tip migration and dislocation in a cadaver model using two catheter designs
}

\author{
Lauren Steffel ${ }^{1}$, Steven K. Howard ${ }^{2}$, Lindsay Borg ${ }^{1}$, Edward R. Mariano ${ }^{2}$, \\ Jody C. Leng ${ }^{2}$, and T. Edward Kim² \\ ${ }^{1}$ Department of Anesthesiology, Perioperative and Pain Medicine, Stanford University School of Medicine, Stanford, \\ ${ }^{2}$ Anesthesiology and Perioperative Care Service, Veterans Affairs Palo Alto Health Care System, Palo Alto, CA, USA
}

\begin{abstract}
Background: New catheter-over-needle (CON) technology for continuous peripheral nerve blockade has emerged, but its effect on the risk of perineural catheter tip dislocation is unknown. Less flexible catheters may be more likely to migrate away from the nerve with simulated patient movement. In the present study, we evaluated catheter tip migration between CON catheters and traditional catheter-through-needle (CTN) catheters during ultrasound-guided short-axis in-plane (SAX-IP) insertion.

Methods: We evaluated the migration of popliteal-sciatic catheters in a prone, unembalmed male cadaver. Thirty catheter placement trials were divided randomly into two groups based on the catheter type: CON or CTN. A single anesthesiology resident placed the catheters by SAX-IP insertion, and the catheters were then examined by ultrasound before and after ipsilateral knee range of motion (ROM) exercises $\left(0^{\circ}-130^{\circ}\right.$ flexion). A blinded expert regional anesthesiologist performed caliper measurements on the ultrasound images before and after the ROM exercises. The primary outcome was the change in distance from the catheter tip to the center of the nerve $(\mathrm{cm})$ between before and after the ROM exercises. Results: The change in the tip-to-nerve distance (median [10th-90th percentile]) was $0.06(-0.16$ to 0.23$) \mathrm{cm}$ for the $\mathrm{CTN}$ catheter and $0.00(-0.12$ to 0.69$)$ for the $\mathrm{CON}$ catheter $(\mathrm{P}=0.663)$. However, there was a statistically significant increase in dislocation out of the nerve compartment for the CON catheter $(4 / 15 ; 0 / 15$ for $\mathrm{CTN})(\mathrm{P}=0.043)$.

Conclusions: Although the use of different catheter designs had no effect on the change in the measured migration distance of popliteal-sciatic catheters, $27 \%$ of the CON catheters were dislocated out of the nerve compartment. These results may influence the choice of catheter design when using SAX-IP perineural catheter insertion.
\end{abstract}

Key Words: Conduction anesthesia, Dislocations, Nerve block, Perineural catheter, Sciatic nerve, Ultrasonography.

Corresponding author: Edward R. Mariano, M.D., M.A.S.

Anesthesiology and Perioperative Care Service, Veterans Affairs Palo Alto Health Care System, 3801 Miranda Avenue (112A), Palo Alto, CA 94304, USA

Tel: 1-650-849-0254, Fax: 1-650-852-3423, Email: emariano@stanford.edu

ORCID: http://orcid.org/0000-0003-2735-248X

Received: July 29, 2016. Revised: September 2, 2016. Accepted: September 13, 2016.

Korean J Anesthesiol 2017 February 70(1): 72-76

https://doi.org/10.4097/kjae.2017.70.1.72

(c) This is an open-access article distributed under the terms of the Creative Commons Attribution Non-Commercial License (http://creativecommons.org/ licenses/by-nc/4.0/), which permits unrestricted non-commercial use, distribution, and reproduction in any medium, provided the original work is properly cited. 


\section{Introduction}

Continuous peripheral nerve block (CPNB) techniques are commonly included as part of multimodal pain management strategies for many surgical procedures [1]. Targeted perineural infusions of local anesthetics have many benefits, including improved pain control with fewer side effects compared with intravenous opioids [2]. However, effective analgesia with perineural catheters is dependent upon accurate tip placement near the target nerve or plexus. Perineural catheter dislocation (movement away from the target nerve) and dislodgement (removal of the catheter from its insertion site) with interruption of analgesia are undesirable and potentially preventable events. A previous study demonstrated an average dislocation rate of $15 \%$ for interscalene and femoral nerve catheters in healthy volunteers performing simulated postoperative activities, and the dislocation rate reached $25 \%$ for femoral nerve catheters over a 6 hour period [3].

The risk factors for perioperative perineural catheter dislocation are unclear, but catheter design may be a contributing factor. While most perineural catheters on the market are catheter-through-needle (CTN) types (e.g., traditional epidural catheters), catheter-over-needle (CON) technology for CPNB has recently emerged. The CON design has been suggested to decrease the risk of catheter tip dislocation based on less fluid leakage around the catheter, resulting in increased stability of catheter dressings [4]. Similar to a peripheral intravenous catheter, the CON perineural catheter has a larger diameter than the insertion needle and may therefore be less likely to become dislodged [5,6]. However, this new catheter design has not been rigorously compared with the established CTN design for ultrasound-guided perineural catheter placement.

We designed this cadaver-based pilot study to test our hypothesis that catheter design affects catheter tip dislocation for popliteal-sciatic perineural catheters inserted using an ultrasound-guided short-axis in-plane (SAX-IP) [7] technique.

\section{Materials and Methods}

With Institutional Review Board exemption and Veterans Affairs Research Committee approval, we evaluated the migration of popliteal-sciatic perineural catheters in a prone, unembalmed human male cadaver weighing $44 \mathrm{~kg}$ and measuring $160 \mathrm{~cm}$ in height.

Thirty catheter placement trials were randomly assigned by a computer-generated randomization sequence (www.randomizer.org) into two groups based on the catheter type (Fig. 1): CON (ON-Q QuikBloc; Halyard Health, Alpharetta, GA, USA) or CTN (Arrow FlexTip Plus; Teleflex Medical, Research Triangle Park, NC, USA). The CON device was a 16-gauge, 76-mm-long catheter designed to fit over a 20-gauge, 100 -mm-long needle
(Fig. 1). The CTN was a 19-gauge, 90-cm-long flexible epidural catheter (Fig. 1) that was modified to enhance tip echogenicity as described previously [8]. A 3-mm segment of a metallic staple (Stanley Bostitch, East Greenwich, RI, USA) was sealed to the distal catheter tip using 2-octylcyanoacrylate glue (Dermabond Advanced; Ethicon, Somerville, NJ, USA).

A human cadaver was placed in the prone position, and a single anesthesiology resident inserted the popliteal-sciatic catheters under ultrasound guidance with a high-frequency linear transducer (HFL50, M-Turbo; Fujifilm SonoSite, Bothell, WA, USA) using the SAX-IP technique [9]. The resident placed each catheter tip anterior to the center of the target nerve within the paraneural sheath [10]. A new puncture site along the lateral thigh was created for each catheter insertion trial. Each catheter was secured by a single-use transparent occlusive dressing after insertion (Bioclusive; Systagenix, Gatwick, West Sussex, UK).

After catheter insertion, the best static ultrasound image of the position of the catheter's tip was determined by the anesthesiology resident and the attending anesthesiologist in the cadaver lab. This image was delivered to a blinded expert regional anesthesiologist to perform caliper measurements of the distance from the catheter tip to the nerve, with secondary confirmation by another blinded expert. The attending anesthesiologist in the cadaver lab then performed five ipsilateral knee range of motion (ROM) exercises $\left(0^{\circ}-130^{\circ}\right.$ flexion) while maintaining the cadaver in the prone position. The same methods for static image capture and caliper measurements were repeated after the ROM exercises.

The primary outcome was the change in distance from the catheter tip to the center of the target nerve between before and after the ROM exercises. The secondary outcome was the proportion of popliteal-sciatic catheters dislocated from the peri-

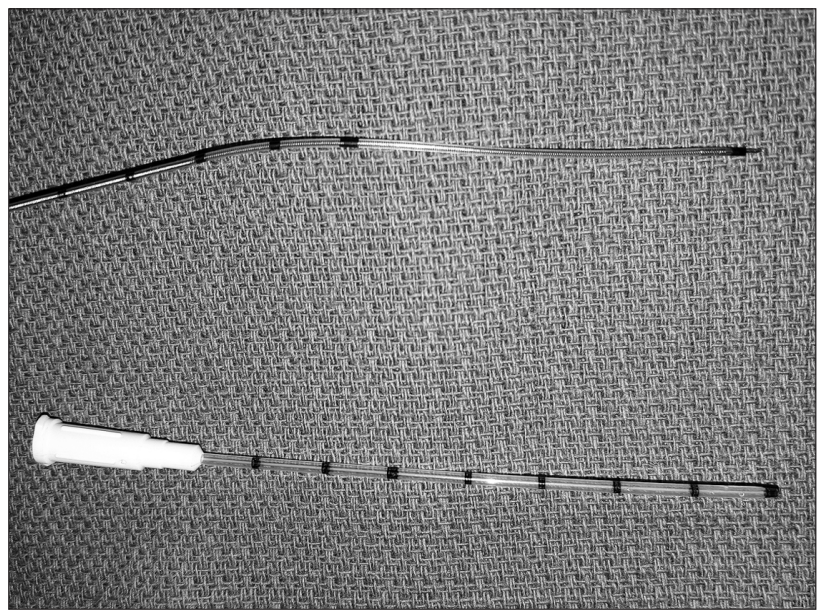

Fig. 1. Image demonstrating the two catheter designs included in this study: flexible epidural catheter-through-needle (top) and catheterover-needle (bottom). 
neural compartment after the ROM exercises.

Because comparative studies of catheter tip migration using this design are lacking, we assumed a catheter stiffness similar to that of the stimulating perineural catheter studied previously [8]. Based on this assumption, $\alpha=0.05$, and $80 \%$ power, we calculated a sample size of 15 trials per group based on the means and standard deviations for the non-tunneled flexible epidural and stimulating catheter groups from the prior study [8] to detect a statistically significant difference in the primary outcome.

The normality of distribution was determined for all scale variables using the Kolmogorov-Smirnov test (NCSS software; NCSS, LLC, Kaysville, UT, USA). For normally distributed data, single comparisons were performed using Student's t-test; for continuous data showing non-normal distributions, the MannWhitney U test was used. The chi-square test or Barnard's exact test ( $\mathrm{n}<5$ in any field) was used to compare categorical data. A $\mathrm{P}<0.05$ was considered statistically significant.

\section{Results}

All 30 catheters were placed successfully using the SAXIP technique on the first attempt; 2 catheters were accidentally dislodged prior to obtaining baseline caliper measurements and were replaced according to their original randomization assignments. All pre- and post-ROM caliper measurements were performed per protocol. The clear occlusive dressings remained intact during and after all ROM trials, and none of the catheters were dislodged from their insertion sites externally. The change in the perineural catheter tip-to-nerve distance was not different between the two groups. The change in distance (median [10th90th percentile]) was $0.06 \mathrm{~cm}(-0.26$ to $0.27 \mathrm{~cm})$ for the CTN catheter and $0.00 \mathrm{~cm}(-0.13$ to $1.01 \mathrm{~cm})$ for the CON catheter $(\mathrm{P}=0.663)$. There was a statistically significant difference in the proportion of popliteal-sciatic catheters that dislocated out of the nerve compartment after ROM: 0/15 (0\%) for CTN versus $4 / 15$ (27\%) for CON ( $\mathrm{P}=0.043)$ (Fig. 2). Of the 4 catheter dislocations, 2 (50\%) occurred in the first 15 trials and 2 (50\%) oc-
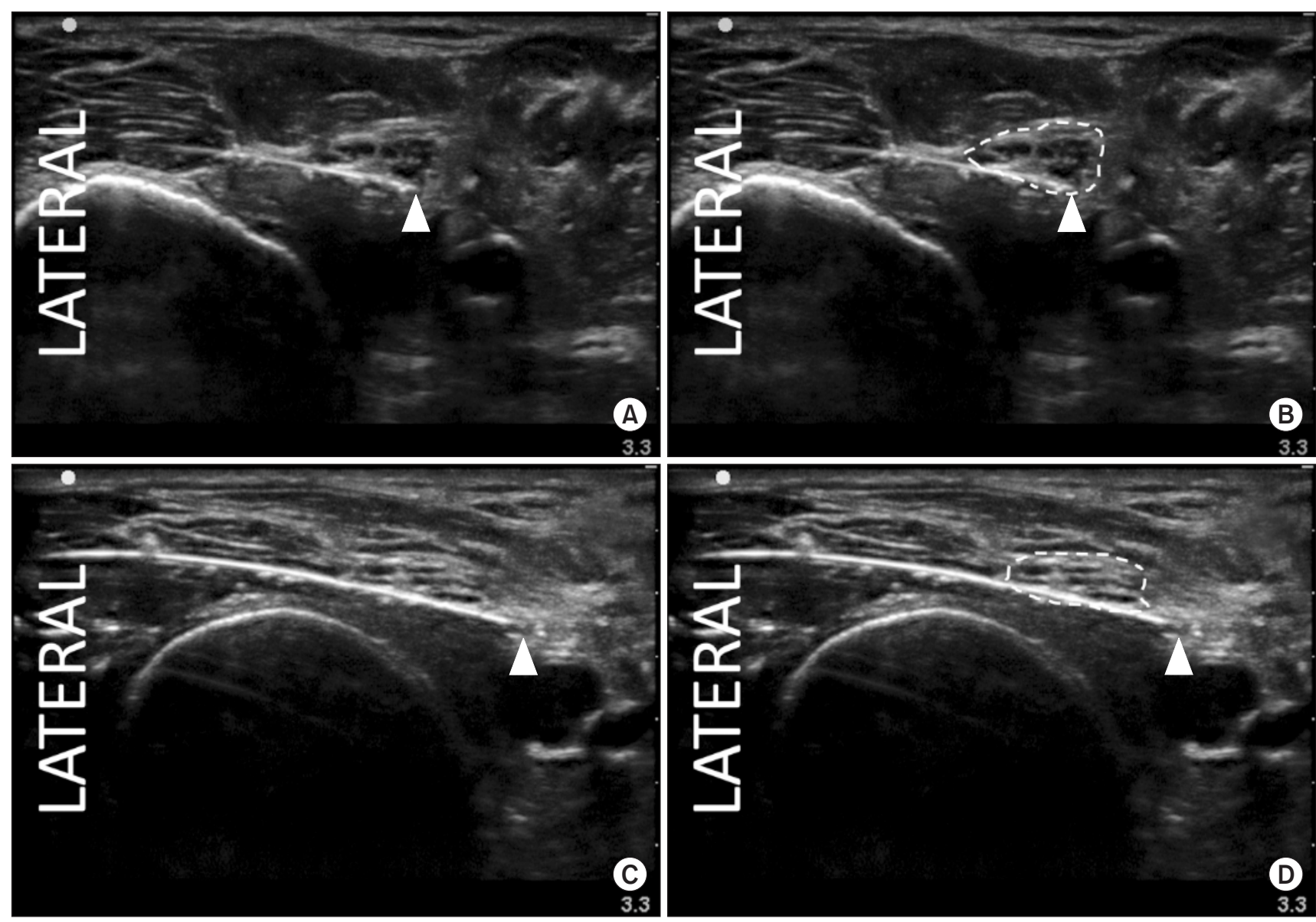

Fig. 2. Sample sonograms demonstrating placement of the catheter-over-needle catheter-over-needle device; panels A and B (same image) show correct positioning of the perineural catheter tip (arrow head) within the perineural compartment (dashed line). Panels C and D (same image) show dislocation of the perineural catheter tip (arrow head) out of the perineural compartment (dashed line). 
curred in the last 15 trials $(\mathrm{P}>0.999)$.

\section{Discussion}

Using an SAX-IP ultrasound-guided technique, the results of this study demonstrate no catheter design-related effect on the measured migration distance of popliteal-sciatic perineural catheters after ROM exercises. However, the greater proportion of catheter tip dislocations (27\%) using the CON device may have important clinical implications. Given the common practice of sending ambulatory foot and ankle patients home with popliteal-sciatic catheters [11-13], regional anesthesiologists may be more inclined to use a more flexible epidural CTN device if they employ an SAX-IP approach to avoid secondary block failure from a dislocated catheter.

For patients with perineural catheters, inadvertent catheter dislocation or complete dislodgement remains a clinically relevant problem, especially in an outpatient setting $[3,14]$. Discharged patients who develop secondary block failure with ensuing severe pain may require care in the emergency department if oral analgesics are insufficient. Patients with functioning perineural catheters at home prefer not to return to the hospital for care related to their catheters and even feel comfortable removing them at home by themselves when their infusions end $[13,15]$. Several strategies for catheter dressing have been suggested to prevent accidental dislodgement, including the application of cyanoacrylate glue at the catheter insertion site [16], subcutaneous tunneling [17], and addition of an adhesive anchoring device $[13,18]$. Tunneling is not mechanically feasible with the CON design, and even the prevention of external dislodgement does not avoid catheter tip dislocation as shown in the present and previously published studies [3,14].

Our results apply exclusively to the SAX-IP perineural catheter insertion technique, which advances the catheter in a perpendicular orientation to the target nerve [7]. This approach using ultrasound guidance has been the most rigorously studied against non-ultrasound techniques $[9,19,20]$ and has been shown to have advantages over certain other ultrasound-guided techniques $[21,22]$. If using a different ultrasound-guided insertion technique [7], such as the short-axis out-of-plane or longaxis in-plane technique, placing the catheter nearly parallel to the target nerve, the effects of patient movement on catheter tip migration and dislocation may differ. Studies involving other CON devices suggest potential benefits to this technology [6,23-
25]. To our knowledge, this is the first study to compare CON with more established flexible CTN devices for SAX-IP ultrasound-guided perineural catheter insertion and demonstrate a difference in catheter dislocation.

There are limitations to the present study. The effects of limb manipulation on catheter migration may differ between cadaver models and living human subjects because of differences in tissue mechanics [26]. However, the use of cadaver models has been described previously in studies of perineural catheter migration [8,27] and has the following advantages: 1) standardization of the catheter procedure and ROM intervention, which is not possible across human subjects, and 2) the ability to alter the flexible epidural catheter to enhance echogenicity [8,27], which occludes the distal orifice and prevents its clinical use for perineural infusion. Due to this modification, fluid was not administered via the catheters; thus, our study was unable to assess leakage. Another potential limitation to this study is the use of the same cadaver for multiple needle and catheter insertions. A new puncture site along the lateral thigh was created for each trial, and there was no increase in the number of catheter dislocations in the later trials compared with the early trials. However, it is possible that subsequent catheter insertion attempts followed previously established tracts, which may have influenced catheter tip migration during the ROM exercises. Furthermore, our results are dependent on the techniques, equipment, and target nerve included in the study design. The use of other ultrasoundguided perineural catheter approaches or other catheter devices may produce different results. Finally, given the limited number of trials in this pilot study, we encourage future research in this area to confirm our findings.

In conclusion, catheter design may influence tip dislocation for popliteal-sciatic perineural catheters placed using an ultrasound-guided SAX-IP technique. Each regional anesthesiologist should carefully consider the choice of catheter design based on his or her preferred insertion approach to minimize the risk of inadvertent tip dislocation and secondary block failure, especially in an ambulatory surgery setting.

\section{Acknowledgments}

The authors gratefully acknowledge the assistance of Mr. Russell Sanchez and the Pathology Service, Veterans Affairs Palo Alto Health Care System, Palo Alto, CA, USA.

\section{References}

1. Chelly JE, Ghisi D, Fanelli A. Continuous peripheral nerve blocks in acute pain management. Br J Anaesth 2010; 105 Suppl 1: i86-96.

2. Richman JM, Liu SS, Courpas G, Wong R, Rowlingson AJ, McGready J, et al. Does continuous peripheral nerve block provide superior pain 
control to opioids? A meta-analysis. Anesth Analg 2006; 102: 248-57.

3. Marhofer D, Marhofer P, Triffterer L, Leonhardt M, Weber M, Zeitlinger M. Dislocation rates of perineural catheters: a volunteer study. Br J Anaesth 2013; 111: 800-6.

4. Ip VH, Rockley MC, Tsui BC. The catheter-over-needle assembly offers greater stability and less leakage compared with the traditional counterpart in continuous interscalene nerve blocks: a randomized patient-blinded study. Can J Anaesth 2013; 60: 1272-3.

5. Tsui BC, Ip VH. Catheter-over-needle method reduces risk of perineural catheter dislocation. Br J Anaesth 2014; 112: 759-60.

6. Tsui BC, Tsui J. Less leakage and dislodgement with a catheter-over-needle versus a catheter-through-needle approach for peripheral nerve block: an ex vivo study. Can J Anaesth 2012; 59: 655-61.

7. Ilfeld BM, Fredrickson M), Mariano ER. Ultrasound-guided perineural catheter insertion: three approaches but few illuminating data. Reg Anesth Pain Med 2010; 35: 123-6.

8. Leng JC, Harrison TK, Miller B, Howard SK, Conroy M, Udani A, et al. A pilot study to assess adductor canal catheter tip migration in a cadaver model. J Anesth 2015; 29: 308-12.

9. Mariano ER, Cheng GS, Choy LP, Loland VJ, Bellars RH, Sandhu NS, et al. Electrical stimulation versus ultrasound guidance for poplitealsciatic perineural catheter insertion: a randomized controlled trial. Reg Anesth Pain Med 2009; 34: 480-5.

10. Andersen HL, Andersen SL, Tranum-Jensen J. Injection inside the paraneural sheath of the sciatic nerve: direct comparison among ultrasound imaging, macroscopic anatomy, and histologic analysis. Reg Anesth Pain Med 2012; 37: 410-4.

11. Capdevila X, Dadure C, Bringuier S, Bernard N, Biboulet P, Gaertner E, et al. Effect of patient-controlled perineural analgesia on rehabilitation and pain after ambulatory orthopedic surgery: a multicenter randomized trial. Anesthesiology 2006; 105: 566-73.

12. Ilfeld BM, Morey TE, Wang RD, Enneking FK. Continuous popliteal sciatic nerve block for postoperative pain control at home: a randomized, double-blinded, placebo-controlled study. Anesthesiology 2002; 97: 959-65.

13. Ilfeld BM, Enneking FK. Continuous peripheral nerve blocks at home: a review. Anesth Analg 2005; 100: 1822-33.

14. Ahsan ZS, Carvalho B, Yao J. Incidence of failure of continuous peripheral nerve catheters for postoperative analgesia in upper extremity surgery. J Hand Surg Am 2014; 39: 324-9.

15. Ilfeld BM, Esener DE, Morey TE, Enneking FK. Ambulatory perineural infusion: the patients' perspective. Reg Anesth Pain Med 2003; 28: 418-23.

16. Klein SM, Nielsen KC, Buckenmaier CC 3rd, Kamal AS, Rubin Y, Steele SM. 2-octyl cyanoacrylate glue for the fixation of continuous peripheral nerve catheters. Anesthesiology 2003; 98: 590-1.

17. Boezaart AP. Continuous interscalene block for ambulatory shoulder surgery. Best Pract Res Clin Anaesthesiol 2002; 16: 295-310.

18. Ilfeld BM. Continuous peripheral nerve blocks in the hospital and at home. Anesthesiol Clin 2011; 29: 193-211.

19. Mariano ER, Loland VJ, Bellars RH, Sandhu NS, Bishop ML, Abrams RA, et al. Ultrasound guidance versus electrical stimulation for infraclavicular brachial plexus perineural catheter insertion. J Ultrasound Med 2009; 28: 1211-8.

20. Mariano ER, Loland VJ, Sandhu NS, Bishop ML, Meunier MJ, Afra R, et al. A trainee-based randomized comparison of stimulating interscalene perineural catheters with a new technique using ultrasound guidance alone. J Ultrasound Med 2010; 29: 329-36.

21. Kim TE, Howard SK, Funck N, Harrison TK, Walters TL, Wagner MJ, et al. A randomized comparison of long-axis and short-axis imaging for in-plane ultrasound-guided popliteal-sciatic perineural catheter insertion. J Anesth 2014; 28: 854-60.

22. Mariano ER, Kim TE, Funck N, Walters T, Wagner MJ, Harrison TK, et al. A randomized comparison of long-and short-axis imaging for in-plane ultrasound-guided femoral perineural catheter insertion. J Ultrasound Med 2013; 32: 149-56.

23. Yu B, Hu X, Zou T, He M, Cai G. Effects of postoperative continuous femoral nerve block analgesia with braun continuous peripheral nerve block catheter set versus novel needle-over-cannula after total knee arthroplasty. Med Sci Monit 2015; 21: 1843-9.

24. Herring AA, Liu B, Kiefer MV, Nagdev AD, Tsui BC. ED placement of perineural catheters for femoral fracture pain management. Am J Emerg Med 2014; 32: 287.e1-3.

25. Ip V, Tsui B. The safety of an interscalene catheter-over-needle technique. Anaesthesia 2013; 68: 774-5.

26. Symons B, Wuest S, Leonard T, Herzog W. Biomechanical characterization of cervical spinal manipulation in living subjects and cadavers. J Electromyogr Kinesiol 2012; 22: 747-51.

27. Webb CA, Kim TE, Funck N, Howard SK, Harrison TK, Ganaway T, et al. Comparison of catheter tip migration using flexible and stimulating catheters inserted into the adductor canal in a cadaver model. J Anesth 2015; 29: 471-4. 